\title{
Excision of an aneurysm of the left atrial appendage
}

\author{
Parsia A. Vagefi, MD, Modassir Choudhry, MD, and Alan D. Hilgenberg, MD, \\ Boston, Massachusetts
}

Supplemental material is available online.

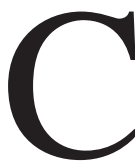

ongenital aneurysmal dilatation of the left atrial appendage is a rare lesion that can commonly be associated with supraventricular arrhythmias, life-threatening systemic embolization, and severe congestive heart failure. ${ }^{1} \mathrm{We}$ report here the case of a 37-year-old man who underwent resection of a giant aneurysm of the left atrial appendage through a limited left thoracotomy without the need for cardiopulmonary bypass (CBP).

\section{Clinical Summary}

A 37-year-old man was told as an adolescent that he had an enlarged heart. He recently sought medical evaluation after reading in the popular press about the death of two young athletes. At the time of presentation he was asymptomatic, and results of his physical examination were unremarkable. An electrocardiogram demonstrated sinus rhythm with frequent premature atrial contractions and left atrial enlargement (Figure E1, a). An echocardiogram showed an echolucent structure measuring $3.6 \times 5.4 \mathrm{~cm}$ adjacent to the base of the left ventricle, with mild extrinsic compression of the ventricle. A chest $\mathrm{x}$-ray film demonstrated a convexity to the left heart border (Figure 1, a). Magnetic resonance imaging showed a left atrial appendage measuring $7.2 \times 9.3 \times 6 \mathrm{~cm}$. A computed tomographic angiogram revealed no evidence of coronary artery disease and confirmed the presence of the aneurysm without evidence of intraluminal thrombus (Figure $1, b$ ). The patient was started on atenolol and warfarin sodium (Coumadin) and was referred for surgery.

The surgical approach was a muscle-sparing, limited left lateral thoracotomy through the fifth intercostal space. A limited longitudinal pericardiotomy revealed an $11-\mathrm{cm}$, thin-walled aneurysm of the left atrial appendage (Figure 2, $a$ ). The aneurysm neck had a normal-sized opening into the left atrium. Intraoperative transesophageal echocardiography confirmed the absence of clot within the aneurysm. An atrial clamp was placed across the base of the

\footnotetext{
From the Division of Cardiac Surgery, Massachusetts General Hospital, Harvard Medical School, Boston, Mass.

Received for publication Nov 10, 2006; accepted for publication Nov 16, 2006.

Address for reprints: Parsia A. Vagefi, MD, Massachusetts General Hospital, 55 Fruit St, GRB-425, Boston, MA 02114-2696 (E-mail: pvagefi@ partners.org).

J Thorac Cardiovasc Surg 2007;133:822-3

$0022-5223 / \$ 32.00$

Copyright $\odot 2007$ by The American Association for Thoracic Surgery doi:10.1016/j.jtcvs.2006.11.025
}

aneurysm at the junction of the left atrial
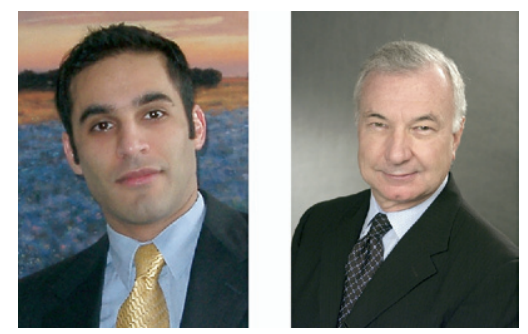

Dr Vagefi and Dr Hilgenberg appendage to the left atrium. Clamping resulted in immediate resolution of the atrial arrhythmia. The aneurysm was excised and the atrial appendage base was closed in two layers with continuous polypropylene suture (Figure 2, $b$ ). The patient was extubated in the operating room, had an uneventful hospital course, and was discharged home on the third postoperative day. At the time of discharge the electrocardiogram demonstrated normal sinus rhythm (Figure E1, b) Pathologic examination demonstrated an atrial wall with marked thinning and fibrosis, consistent with an aneurysm of the atrial appendage.

\section{Discussion}

Multiple surgical approaches have been used for management of aneurysms of the left atrial appendage, including median sternotomy with or without $\mathrm{CPB},{ }^{1-3}$ left thoracotomy, ${ }^{1}$ and minimally invasive endoscopic resection. ${ }^{4}$ It has been suggested that aneurysmectomy aided by CPB is a safer surgical method for this anomaly. ${ }^{2}$ However, in the absence of a broad-based aneurysm neck or thrombus within the aneurysm sac, both of which conditions would require extracorporeal circulation, a lateral thoracotomy without CPB can be used for resection. The latter is a less invasive open approach with improved exposure, because it allows for entry into the chest directly overlying the aneurysm. In contrast, medial retraction of the pulmonary artery for clamp application is required through a median sternotomy. ${ }^{1}$ Based on their historical use for closure of the nonaneurysmal left atrial appendage, surgical staplers have been used for aneurysm resection to minimize the cardiac manipulation required to gain exposure through a median sternotomy. ${ }^{3}$ However, when resection is carried out in the absence of CPB, given the thinned, fragile walls of these aneurysms, our preference is for clamp application with full excision and oversewing in lieu of stapling. Finally, although minimally invasive endoscopic resection has been previously reported for aneurysm resection, ${ }^{4}$ the latter entails an overly complicated surgical approach that still requires the need for both $\mathrm{CPB}$ and a minithoracotomy.

The diagnosis of aneurysms of the left atrial appendage can be suggested by radiologic examination consisting of a plain chest $\mathrm{x}$-ray film. However, preoperative assessment of the location of the aneurysm in relation to the coronary arteries, as well as the pulmonary veins, can be visualized with magnetic resonance imaging or computed tomography. ${ }^{5}$ Furthermore, preoperative cardiac computed tomographic scan can evaluate potential clot burden within the aneurysm, as well as assess atherosclerotic changes in the coronary arteries, obviating the need for cardiac angiography. When a less invasive surgical approach is being used, such as a limited lateral thoracotomy, transesophageal echocardiography can serve as a useful 

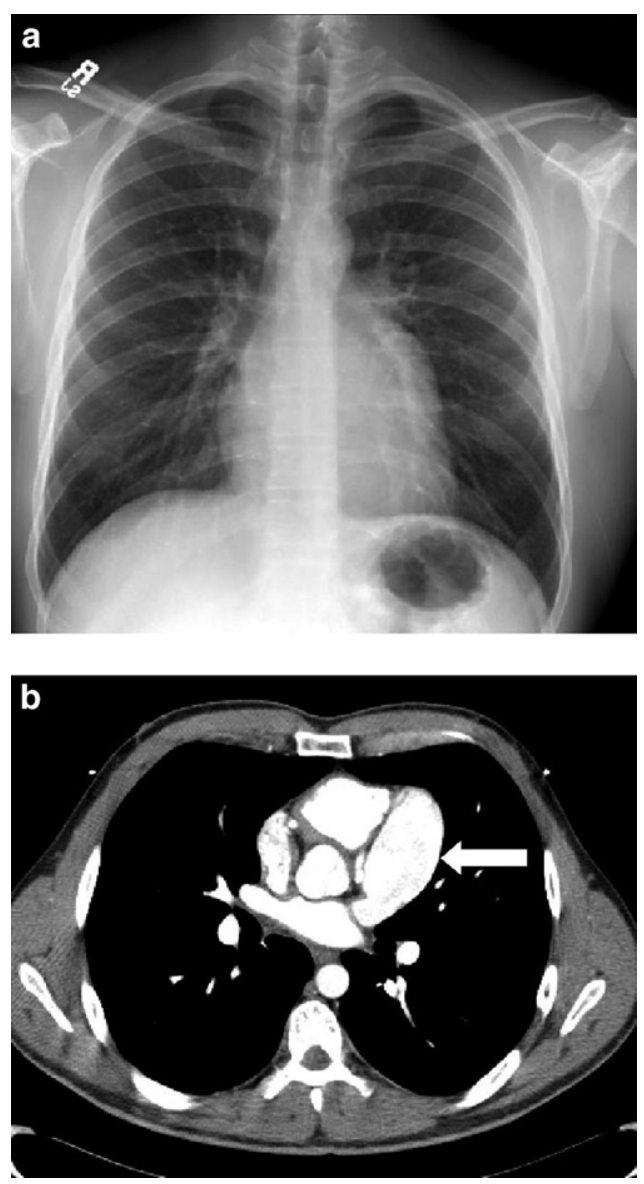

Figure 1. a, Posteroanterior film of the chest demonstrated a slight convex bulge to the upper portion of the left heart border. b, Three-dimensional computed tomographic angiogram of the chest demonstrated a large aneurysm of the left atrial appendage with no thrombus.

intraoperative adjunct by further delineating the relevant anatomy in real time. ${ }^{3}$

Resection of an aneurysm of the left atrial appendage through a limited left thoracotomy allows for a simplified, safe approach to aneurysm resection that avoids the need for CBP.
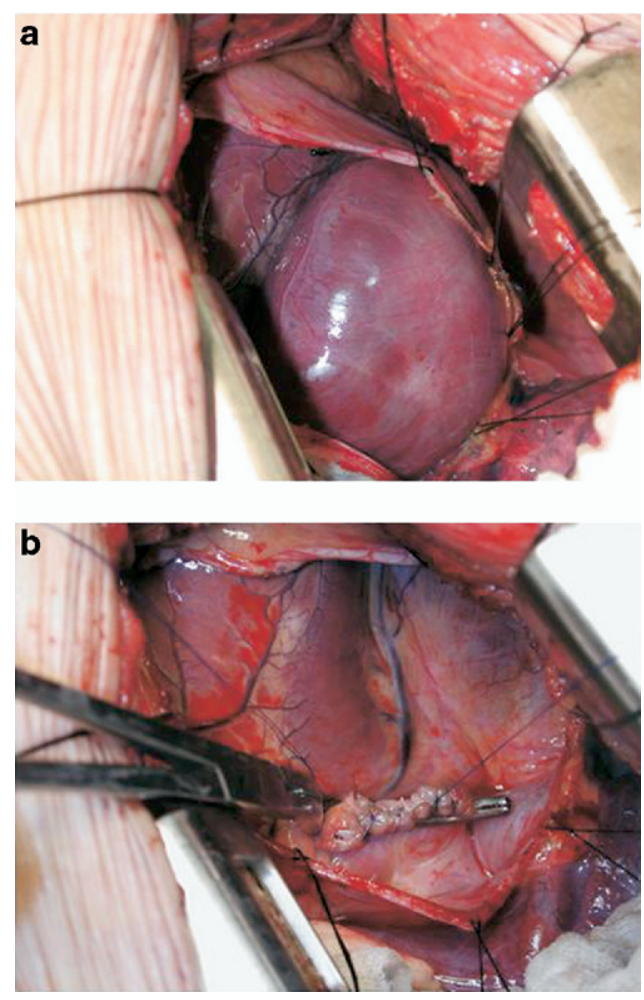

Figure 2. Aneurysm of the left atrial appendage after opening of the pericardial sac (a) and after surgical excision (b).

\section{References}

1. Zhao J, Ge Y, Yan H, Pan Y, Liao Y. Treatment of congenital aneurysms of the left atrium and left atrial appendage. Tex Heart Inst $J$. 1999;26:136-9.

2. Pomerantzeff PM, Freyre HM, de Almeida Brandao CM, Pereira Barreto AC, Almeida de Oliveira S. Aneurysm of the left atrial appendage. Ann Thorac Surg. 2002;73:1981-3.

3. Burke RP, Mark JB, Collins JJ, Cohn LH. Improved surgical approach to left atrial appendage aneurysm. J Card Surg. 1992;7:104-7.

4. Kiaii B, Doll N, Kuehl M, Mohr FW. Minimally invasive endoscopic resection of a giant left atrial appendage aneurysm. Ann Thorac Surg. 2004;77:1437-8.

5. Hoffmann U, Hamed N, Herold C, Globits S. Radiological signs of a left atrial aneurysm. Eur Radiol. 2000;10:1332-4. 


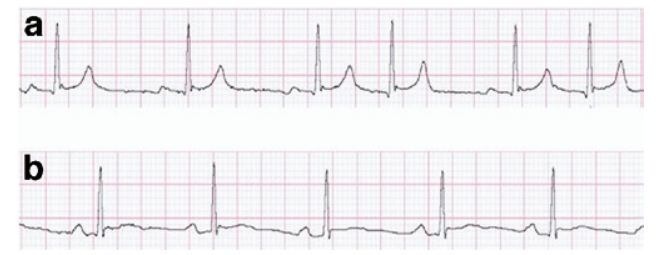

Figure E1. a, Preoperative electrocardiogram (lead II). b, Postoperative electrocardiogram (lead II). 